\title{
Streptococcus oralis Meningitis
}

\author{
$\underline{\text { João Cruz Cardoso }}{ }^{1}$, David Ferreira ${ }^{2}$, Rui Assis ${ }^{3}$, Joana Monteiro $^{3}$, Inês Coelho ${ }^{3}$, André Real ${ }^{3}$, Nuno Catorze ${ }^{3}$ \\ ${ }^{1}$ Internal Medicine Department, Hospital de Santarém EPE, Santarém, Portugal \\ 2Internal Medicine Department, Centro Hospitalar Médio Tejo EPE, Abrantes, Portugal \\ ${ }^{3}$ Critical Care Medicine Department, Centro Hospitalar Médio Tejo EPE, Abrantes, Portugal
}

\section{Doi: 10.12890/2021_002349- European Journal of Case Reports in Internal Medicine - 다 EFIM 2021}

Received: 02/02/2021

Accepted: 20/04/2021

Published: 07/05/2021

How to cite this article: Cruz Cardoso J, Ferreira D. Assis R, Monteiro J, Coelho I, Real A, Catorze N. Streptococcus oralis meningitis. EJCRIM 2021;8: doi:10.12890/2021_002349.

Conflicts of Interests: The authors disclose no conflicts of interest.

This article is licensed under a Commons Attribution Non-Commercial 4.0 License

\section{ABSTRACT}

Streptococcus oralis is part of the normal flora of the oropharyngeal, nasal, gastrointestinal and genitourinary tracts. Classically, it shows low pathogenicity and virulence, but can very rarely cause meningitis in patients who have undergone dental procedures and have poor oral hygiene.

The purpose of this report is to highlight the importance of considering S. oralis as a cause of meningitis in patients with poor oral hygiene. A 53-year-old man was admitted to the emergency department with high fever $\left(39.4^{\circ} \mathrm{C}\right)$, headache and drowsiness. His mouth was unhygienic. He was diagnosed with meningitis and empiric antibiotics (ceftriaxone plus ampicillin) and dexamethasone were started. S. oralis was isolated from cerebrospinal fluid. Ampicillin and dexamethasone were stopped, while ceftriaxone was continued with full recovery of the patient.

\section{LEARNING POINTS}

- Viridans streptococci such as Streptococcus oralis can rarely cause meningitis as well as endocarditis in patients with poor dental hygiene.

- Streptococcus viridans meningitis responds well to empiric antibiotic therapy.

- Patients with any form of streptococcus viridans infection should be screened for endocarditis.

\section{KEYWORDS}

Streptococcus oralis, meningitis, oral hygiene

\section{INTRODUCTION}

Streptococcus oralis, a member of the Streptococcus mitis family, belongs to the viridans group and is part of the normal flora of the oropharyngeal, nasal, gastrointestinal and genitourinary tracts ${ }^{[1]}$. Classically, it shows low pathogenicity and virulence but is an important cause of subacute bacterial endocarditis ${ }^{[2]}$. Although viridans streptococcus often causes bacteriaemia ${ }^{[3]}$ and can occur as a pathogenic microorganism in immunocompromised patients ${ }^{[4]}$, its association with meningitis is uncommon; the viridans streptococci (including $S$. oralis) account for only $0.3-2.4 \%$ of all causes of meningitis ${ }^{[3]}$.

A review of the literature showed that only eight cases of $S$. oralis meningitis have ever been described and were associated with dental procedures, spinal anaesthesia, malignancy, cerebrospinal fluid leak and gingival bleeding ${ }^{[1]}$.

We describe a case of meningitis caused by S. oralis associated with alcoholism and poor oral hygiene. 


\section{CASE DESCRIPTION}

A 53-year-old man was admitted to the emergency department of our hospital due to altered mental status. His medical history included hypertension, dyslipidaemia and alcoholism. He presented with a 4-day history of high fever $\left(39.4^{\circ} \mathrm{C}\right.$ ), headache and drowsiness (Glasgow Coma Scale score 13). A neurological examination revealed neck stiffness but no evidence of Kernig's or Brudzinski's signs. He had poor oral hygiene including dental caries and tongue plaque. His neck stiffness, altered mental status and fever were consistent with meningitis. Laboratory tests revealed the following: haemoglobin $13.3 \mathrm{~g} / \mathrm{dl}$, haematocrit $38.6 \%$, white blood cell count $22.26 \times 10^{\%} / 1(89.7 \%$ neutrophils, $2.4 \%$ lymphocytes and $7.7 \%$ monocytes), platelet count $377 \times 10^{\%} / \mathrm{l}$, glucose level $150 \mathrm{mg} / \mathrm{dl}$ and C-reactive protein $24.24 \mathrm{mg} / \mathrm{dl}$. Renal and hepatic function and coagulation tests were normal. A computed tomography scan of the brain showed discreet prominence of the ventricular system, suggesting the presence of hydrocephalus in the context of meningitis. A lumbar puncture was performed and revealed cloudy cerebrospinal fluid (CSF); analysis showed pleocytosis with predominant polynuclear cells $\left(295 / \mathrm{mm}^{3} ; 70 \%\right.$ polynuclear, $30 \%$ mononuclear) as well as increased levels of protein $(700 \mathrm{mg} / \mathrm{dl})$ and low levels of glucose $(1 \mathrm{mg} / \mathrm{dl})$. Sets of anaerobic and aerobic blood cultures were obtained and cerebrospinal fluid was sent for culture. A transthoracic echocardiogram was negative for infectious endocarditis.

Empiric meningitis antibiotic treatment for patients over 50 years of age was initiated: ceftriaxone $4 \mathrm{~g} /$ day and ampicillin $12 \mathrm{~g} /$ day, in addition to dexamethasone $8 \mathrm{mg}$ every 6 hours. Chlorhexidine mouthwashes were performed three times a day. On day 1, a Gram-positive coccus grew on CSF and S. oralis was isolated on day 2; all sets of blood cultures were negative. The isolate was susceptible to penicillin and ceftriaxone, so the ampicillin was suspended and dexamethasone stopped. The patient's symptoms, such as fever and disturbance of consciousness, slowly improved. The oral medicine department was consulted and was asked to treat the patient's teeth. By day 3 , he had fully recovered and was transferred to the medical ward.

\section{DISCUSSION}

Meningitis caused by viridans streptococci can occur in patients of all ages. The results of spinal fluid analysis are similar to the findings in other types of pyogenic meningitis ${ }^{[3]}$. Bacterial meningitis is a potentially fatal disease and delayed empiric antibiotic treatment increases the risk of permanent brain damage ${ }^{[1]}$.

In parallel with meningitis treatment, infectious endocarditis should be ruled out because $S$. oralis can cause this condition ${ }^{[1]}$. We evaluated our patient for the presence of infective endocarditis but echocardiography showed that there was no vegetation or valve disease.

Dental caries and periodontal disease should be treated as most cases of streptococcal endocarditis are caused by poor oral hygiene rather than by dental procedures ${ }^{[5]}$. However, it is unclear if good oral hygienic can prevent $S$. oralis meningitis as so few cases have been reported ${ }^{[2]}$.

Poor oral hygiene and dental treatment have a considerable role in S. oralis bacteraemia and in endocarditis ${ }^{[2]}$. In our opinion, despite the patient's blood culture being negative, and assuming that the mechanism is similar, the poor oral hygiene was the probable cause of meningitis in this patient. However, in a third of such meningitis cases, the exact source of infection is not clear ${ }^{[3]}$.

To our knowledge, this is the ninth reported case of $S$. oralis meningitis ${ }^{[2]}$ and the first seen in Portugal. Although $\mathrm{S}$. oralis is believed to be a rare cause of meningitis, it should be considered in the differential diagnosis of meningitis in alcoholic patients with poor oral hygiene. Clinical examination of the oral cavity in patients diagnosed with meningitis is very important.

\section{REFERENCES}

1. Patel K, Memon Z, Prince A, Park C, Sajan A, Ilyas N. Streptococcus oralis meningitis from right sphenoid meningoencephalocele and cerebrospinal fluid leak. BMC Infect Dis 2019;19(1):960.

2. Nakamura Y, Uemura T, Kawata Y, Hirose B, Yamauchi R, Shimohama S. Streptococcus oralis meningitis with gingival bleeding in a patient: a case report and review of the literature. Intern Med 2021;60(5):789-793.

3. Montejo M, Aguirrebengoe K. Streptococcus oralis meningitis after dental manipulation. Oral Surg Oral Med Oral Pathol Oral Radiol Endod 1998;85(2):126-127.

4. Chang WN, Wu JJ, Huang CR, Tsai YC, Chien CC, Lu CH. Identification of viridans streptococcal species causing bacterial meningitis in adults in Taiwan. Eur J Clin Microbiol Infect Dis 2002;21(5):393-396.

5. Eykyn SJ. Infective endocarditis: some popular tenets debunked? Heart 1997;77(3):191-193. 